\title{
Leaching Behavior of a Fluorescent Pyoverdin Immobilized in Sol-Gel Glass
}

\author{
Michael F. Yoder and William S. Kisaalita*
}

Cellular Bioengineering Laboratory, Faculty of Engineering, University of Georgia, Athens, GA 30602, USA

\begin{abstract}
The leaching behavior of fluorescent siderophore, pyoverdin (iron biosensor), immobilized in acid-catalyzed sol-gel glass is examined. By varying the $\mathrm{R}$ value (molar ratio of water:silicon), three different formulations of pyoverdindoped sol-gel glass pellets $(\mathrm{R}=5.6,8.2,10.8)$ were made with tetramethyl orthosilicate (TMOS). At three different aging times (2, 4, and 6 weeks), 7-day leaching experiments were conducted on whole and ground pellets. Two different leaching solutions were used: $0.1 \mathrm{M}$ acetate buffer, $\mathrm{pH}$ 5.0, and $1 \mathrm{M} \mathrm{HCl}$. Pyoverdin immobilization resulted in conformation changes as suggested by the appearance of two emission peaks centered at 440 and $505 \mathrm{~nm}$. As expected, pyoverdin leached more rapidly from the ground glass; $61 \%$ of the pyoverdin leached from the ground glass within the first 20 minutes, while after 3 hours, only $54 \%$ of the pyoverdin had leached from the whole pellets. As the sol-gel glass aged from 2 to 6 weeks, the initial fluorescence of the ground glass decreased by $34-46 \%$ for the three sol-gel formulations, and the 7-day cumulative leachate decreased by $13-31 \%$. However, sol-gel with the lowest R value (5.6) retained pyoverdin better that sol-gels at higher $\mathrm{R}$ values. The release of pyoverdin was also characterized by the diffusional exponent (n), which ranged from 0.527 to 0.802 for the ground glass, and from 0.276 to 0.415 for the whole pellets, confirming that whole pellets retained pyoverdin better than ground glass. Taken together, the results suggest for a given sensor configuration, retention of pyoverdin can be optimized with respect to pellet size, $R$ value and aging time.
\end{abstract}

Key Words: Biosensor, fluorescent, leaching, pyoverdin, sol-gel.

\section{INTRODUCTION}

Porous glass produced by the sol-gel process [1] continues to gain popularity as an immobilization matrix for biosensors. More researchers are recognizing the ease with which sol-gel glass can be made, the wide variety of molecules that can be immobilized in it, and the wide variety of biosensor applications for it [2-5]. But, for sol-gel based biosensors to become a commercial reality, not only must they be specific and sensitive for the analyte being measured, they must also be stable [6]. Depending upon the environmental conditions the biosensor is exposed to, over time there can be changes in biosensor performance. This may be due to: conformational changes of the entrapped biomolecule [7]; a decrease in sol-gel matrix pore size [4]; leaching of the biomolecule out of the sol-gel matrix [8]; or leaching of a part of the biomolecule [9].

A doped sol-gel glass can be made in many different ways by varying the following: 1. silicon precursor used (such as tetramethyl orthosilicate (TMOS) and tetraethyl orthosilicate (TEOS); 2. additives (alcohol, surfactant, polymer, etc.); 3. pH; 4. buffer type and concentration; 5. molar ratio of water:silicon ( $\mathrm{R}$ value); 6 . acid or base catalyst; 7 . at what stage components are added; 8 . aging conditions (wet or dry, and temperature). All of these variables, along with the properties and concentration of the dopant, can affect the physical and chemical properties of the doped sol-gel glass.

*Address correspondence to this author at the Cellular Bioengineering Laboratory, Faculty of Engineering, University of Georgia, Athens, GA 30602, USA; Tel: (706) 542-0835; Fax: (706) 542-8806;

E-mail: williamk@engr.uga.edu
When biomolecules or indicator molecules have been doped into TMOS and TEOS sol-gel glasses, a wide variety of leaching behaviors have been reported $[8,10,11]$.

In a pioneering study, Barrero et al. [12] immobilized pyoverdin in a TMOS sol-gel glass to produce a sensitive ferric biosensor. Pyoverdin is a fluorescent siderophore produced by Pseudomonas bacteria growing in iron-low media (also spelled pyoverdine [13]). Also, Yoder and Kisaalita [14] studied the iron specificity of pyoverdin (from $P$. aeruginosa) that was immobilized in a TMOS sol-gel glass. In both of these studies no detailed leaching results were reported. Barrero et al. [12] reported that there was no leaching, but as the biosensor was repeatedly used the fluorescent intensity baseline kept decreasing.

The long-term goal motivating this research is the development of a high-throughput iron biosensor based on pyoverdin immobilized in sol-gel glass. Because leaching of pyoverdin was observed in preliminary experiments, the objective of this paper was to characterize the leaching of pyoverdin from acid-catalyzed TMOS sol-gel glass. This study evaluated the effect of the following four variables on the leaching of pyoverdin: 1 . the size of the doped sol-gel glass (ground, sieved pieces or whole pellets); 2 . three R values $(5.6,8.2$, and 10.8); 3 . how long the doped sol-gel glass had aged $(2,4,6$ weeks) before testing for leaching; and 4. the $\mathrm{pH}$ of the leaching solution (acetate buffer, $\mathrm{pH} 5.0$ or hydrochloric acid, $\mathrm{pH} 0.02$ ).

At different ages of curing, pyoverdin-doped sol-gel glass was soaked for 7 days in leaching solution. For each 
7-day leaching experiment, fluorescence emission spectra were recorded for both the immobilized pyoverdin and leached pyoverdin. Comparisons of the spectra and fluorescent intensity are made, and the diffusional exponent, $\mathrm{n}$ [15] was calculated to compare leaching rates.

\section{MATERIALS AND METHODS}

Fluorescence measurements (of samples in 96-well plates) were made with a FLEXstation ${ }^{\mathrm{TM}}$ benchtop scanning fluorometer (Molecular Devices Corp., Sunnyvale, CA). The fluorescence measurements were done in the bottom read mode, temp. $=25^{\circ} \mathrm{C}$, PMT (photomultiplier tube) sensitivity $=$ high, with no cutoff filters, and fluorescence was recorded in relative fluorescence units (rfu). For emission scans, the excitation (exc.) was set to $370 \mathrm{~nm}$ and the emission (emis.) was scanned from $390-600 \mathrm{~nm}$, in $5 \mathrm{~nm}$ increments. Pyoverdin solutions, pyoverdin-doped sol-gel glass (ground), and the leachates from the experiments were placed in Corning Costar® 96-well plates (no. 3631, polystyrene, black with clear, flat bottom).

\subsection{Solutions}

Tetramethyl orthosilicate (TMOS) was 98\% (Aldrich). Deionized (d.i.) water was ultrapure (Barnstead, Nanopure Infinity). Methanol was 99.9 \%, HPLC grade (Fisher). The $0.1 \mathrm{M}$ acetate buffer, $\mathrm{pH} 5.0$, was prepared with $74.0 \mathrm{ml} 0.2$ $\mathrm{M}$ acetic acid $+176.0 \mathrm{ml} 0.2 \mathrm{M}$ sodium acetate $+250.0 \mathrm{ml}$ d.i. water, for a total volume of $500 \mathrm{ml}$.

\subsection{Pyoverdin}

Pyoverdin from Pseudomonas aeruginosa ATCC 15692 was produced and purified by copper-chelate chromatography, according to the procedures of Yoder and Kisaalita [14]. Pyoverdin from $P$. aeruginosa consists of an octapeptide, containing both $\mathrm{D}$ - and L-amino acids and a chromophore derived from 2,3-diamino-6,7-dihydroxyquinoline [16]. The purified pyoverdin solution (in deionized water) was previously determined to be $6.0 \mathrm{mM}$ [14]. The same stock was used for all experiments.

\subsection{Preparation of Pyoverdin-Doped Sol-Gel Glass}

Three formulations of sol-gel glass pellets were made with a single-step, acid-catalyzed procedure, very similar to that used by Yoder and Kisaalita [14]. With continuous stirring, in the order listed, the following solutions were added to a $100 \mathrm{ml}$ glass beaker: $9.6 \mathrm{ml}$ methanol, $7.0 \mathrm{ml} 2 \mathrm{~N}$ nitric acid, $0-6.0 \mathrm{ml}$ deionized water, $65.1-80.0 \mu \mathrm{l}$ of purified pyoverdin solution, and $9.6 \mathrm{ml}$ TMOS. The amount of added water $(0,3.0$, or $6.0 \mathrm{ml})$ was varied to achieve $\mathrm{R}$ values of $5.6,8.2,10.8$, in the three formulations, hereafter referred to as $\mathrm{A}, \mathrm{B}, \mathrm{C}$, respectively. The amount of pyoverdin added to each formulation (A, B, C) was varied $(65.1,72.8,80.0 \mu 1$, respectively) so that the ratio of pyoverdin to the total volume of sol-gel solution was constant. The solution was stirred for an additional minute, and then $80 \mu \mathrm{l}$ of the sol-gel solution was placed into each microwell of a 96-well plate (Falcon®, \#3072, polystyrene, clear, flat bottom) and the lid was sealed onto the plate with tape. After 7 days, the tape was removed, and after 12 days the lid was tipped to allow more rapid drying. The plate was weighed every few days to see when the sol-gel pellets had reached constant weight. Preparation and aging of the pellets was done at room temperature (ca. $22^{\circ} \mathrm{C}$ ). Control sol-gel glass pellets, without pyoverdin, were also made for each formula, with deionized water being used in place of the pyoverdin solution.

Leaching experiments, each 7 days long, were conducted at approximately 2, 4, and 6 weeks after the sol-gel pellets were made on day 0 . The 2 -week experiments were started on day 17 , the 4-week experiments were started on day 31 , and the 6-week experiments were started on day 45 .

\subsection{Spectra of Pyoverdin Solutions}

Solutions of $5.0 \mu \mathrm{M}$ pyoverdin were made in both $0.1 \mathrm{M}$ acetate buffer $(\mathrm{pH} \mathrm{5.0)}$ ) and $1.0 \mathrm{M} \mathrm{HCl}$. For each pyoverdin solution, 8 microwells of a Costar 96-well plate were filled with $200 \mu \mathrm{l}$, and the emission (exc. $370 \mathrm{~nm}$ ) and excitation spectra (emis. $455 \mathrm{~nm}$ ) were measured and averaged for the 8 microwells. Spectra of control solutions (acetate buffer and $\mathrm{HCl}$ without pyoverdin) were measured in the same way. The control spectra were subtracted from the spectra for pyoverdin solutions, and plots were made of these corrected spectra.

\subsection{Leaching Experiment with Ground and Sieved Sol- Gel Glass}

In preliminary experiments, pyoverdin-doped sol-gel glass was ground and placed in the wells of a 96-well (clear bottom) plate with acetate buffer $(\mathrm{pH} \mathrm{5.0)}$. The addition of iron resulted in fluorescence quenching of the immobilized pyoverdin. Approximately $0.0156 \mathrm{~g}$ of the ground sol-gel glass was enough to fully cover the bottom of each well, and by sieving it between fine mesh screens the variation in fluorescence measurements was kept low.

For the 3 formulations of pyoverdin-doped sol-gel glass and the 3 control sol-gel glasses, at the aging times of 2, 4, and 6 weeks, the following protocol was followed.

Sol-gel pellets were weighed and then ground between 2 glass beakers. The pellets were placed in a $250 \mathrm{ml}$ Pyrex ${ }^{\circledR}$ (heavy duty) beaker, and a $180 \mathrm{ml}$ Pyrex ${ }^{\circledR}$ (tall form) beaker was placed inside this to crush the pellets with hand pressure. The ground glass was sieved between $1.0 \mathrm{~mm}$ and 0.50 mm nylon screen cloth (Small Parts Inc., Miami Lakes, FL) and then 8 portions of approximately $0.0156 \mathrm{~g}$ each were placed into 8 wells of a flat bottom, 96-well plate (Costar®), black with clear bottom).

To each of these 8 wells, $200 \mu \mathrm{l}$ of $0.1 \mathrm{M}$ acetate buffer $(\mathrm{pH}$ 5.0) was added at time $=0$. At 3 minutes, the initial fluorescence of the glass was measured (exc.370 nm, emis. scanned from $390-600 \mathrm{~nm}$ ). At 10 minutes, the buffer solution (hereafter called leachate) was transferred to empty wells in the same 96-well plate. Fresh acetate buffer $(200 \mu 1$ per well) was added to the glass and the fluorescence was immediately measured before any more pyoverdin could leach out. The fluorescence of the leachate was then measured. This same procedure was followed at times $20 \mathrm{~min}$., 1 hr., 3 hrs., 10 hrs., 24 hrs., 3 days, and 7 days on the same glass samples. This protocol was also followed for the control sol-gel glasses (without pyoverdin) to have background fluorescence measurements. 


\subsection{Leaching Experiment with whole Pellets of Sol-Gel Glass}

For the 3 formulations of pyoverdin-doped sol-gel glass and the 3 control sol-gel glasses, at the aging times of 2, 4, and 6 weeks, the following protocol was followed. About $0.234 \mathrm{~g}$ (between $10^{1 / 2}-13^{1 / 2}$ pellets) of whole glass pellets were weighed and placed in a $15 \mathrm{ml}$ centrifuge tube (Corning, \#430052, polypropylene). At time $=0,3.0 \mathrm{ml}$ of $0.1 \mathrm{M}$ acetate buffer ( $\mathrm{pH}$ 5.0) was added to each tube. This ratio of $0.234 \mathrm{~g}$ glass: $3.0 \mathrm{ml}$ solution was the same ratio as that used for ground sol-gel glasses (0.0156 g glass:200 $\mu$ l solution). At $10 \mathrm{~min}$., the leachate was removed and transferred to a separate polypropylene tube, and $3.0 \mathrm{ml}$ of fresh buffer was added to the glass pellets. Two portions of $200 \mu 1$ of the leachate were transferred to wells in a 96-well plate and the fluorescence was measured. This same procedure was followed at times 20 min., 1 hr., 3 hrs., 10 hrs., 24 hrs., 3 days, and 7 days. Preliminary experiments demonstrated the presence of scattering artifacts when whole glass pellets were placed in the wells of a 96-well plate. Therefore, the fluorescence of the whole glass pellets was not measured.

\subsection{Leaching Experiments with $1 \mathrm{M} \mathrm{HCl}$}

Barrero et al. [12] used $1 \mathrm{M} \mathrm{HCl}$ to remove iron bound to pyoverdin immobilized in sol-gel glass. Yoder and Kisaalita [14] also used this method to regenerate their iron biosensor so it could be repeatedly used for iron quenching assays. To study the effect of $\mathrm{HCl}$ on the leaching of pyoverdin, after the sol-gel glass had aged 4 weeks, leaching experiments with ground sol-gel glass and with whole pellets were conducted as previously described, but with $1.0 \mathrm{M} \mathrm{HCl}$ as the leaching solution.

\subsection{Data Analysis for the Leaching Experiments with Ground Sol-Gel Glass}

Data reduction for the emission spectra (exc. $370 \mathrm{~nm}$ ) of both the immobilized pyoverdin and the leachates consisted of first selecting the emission at $450 \mathrm{~nm}$, as this was near the maximum emission peak for all readings. Then, to correct for differences in the aged pellets and differences in the control sol-gel glasses, an adjusted fluorescence value (described below) was calculated.

Although the 3 formulations of pyoverdin-doped pellets were made with the same ratio of pyoverdin to total volume of sol-gel solution, because the aged pellets dried different amounts, the ratio of pyoverdin to weight of dried sol-gel glass varied slightly between the 3 formulations. Also, the spectra of the control sol-gel glasses showed some backgroung fluorescence and scattering due to the sol-gel matrix. Thus, to properly compare the fluorescence (exc./emis. 370/ $450 \mathrm{~nm}$ ) measurements (in relative fluorescence units, $\mathrm{rfu}$ ) the fluorescence per nanomole of immobilized pyoverdin (here called adjusted fluorescence) was calculated as follows:

$$
\text { FLadj }=(\text { FLpvd }- \text { FLc }) /(\text { Mpvd } \times \text { Wt })
$$

where: FLadj is the adjusted fluorescence (rfu per nanomole immobilized pyoverdin); FLpvd is the fluorescence ( $\mathrm{rfu}$ ) of pyoverdin-doped sol-gel glass; FLc is the fluorescence (rfu) of control sol-gel glass; Mpvd is the nanomoles pyoverdin per gram of sol-gel glass; Wt is the weight ( $\mathrm{g}$ ) of pyoverdindoped sol-gel glass.
FLpvd, FLc, and Wt were mean values per microwell, each calculated from 8 microwells, and the corresponding standard deviations were input into an equation to calculate the standard deviation of the adjusted fluorescence. Mean values of adjusted fluorescence (exc./emis. 370/450 nm) were calculated for: 1) the initial fluorescence of the pyoverdin-doped sol-gel glass; 2) the fluorescence of the pyoverdin-doped sol-gel glass after 7 days of leaching; and 3 ) the cumulative (over 7 days) fluorescence of the leachates. These means were compared with multiple t-tests (calculated in Microsoft Excel). Means that were not significantly different $(\alpha=0.05)$ were given the same letter designation.

\subsection{Data Analysis for Whole Pellet Leachates}

The fluorescence (exc./emis. 370/450 nm) values for the whole pellet leachates were adjusted as described for the ground sol-gel glass, except Wt in equation (1) was the weight of sol-gel glass per $200 \mu \mathrm{l}$ buffer. This allowed direct comparison to the (adjusted) fluorescence values determined for the ground sol-gel glass experiments.

\subsection{Leaching rate Determination}

The following simple equation was developed by Ritger and Peppas [15] to describe the solute (drug) release from non-swellable, controlled release polymers:

$$
\mathrm{M}_{\mathrm{t}} / \mathrm{M}_{\infty}=\mathrm{k} \mathrm{t}^{\mathrm{n}}
$$

where $M_{t}$ is the mass of drug released at time $t, M_{\infty}$ is the mass of drug released as time approaches infinity, $\mathrm{k}$ is a constant (which depends on the characteristics of the polymer matrix and the doped drug), and $\mathrm{n}$ is the diffusional exponent. When this equation is applied to data of the first $60 \%$ of the fractional release $\left(M_{t} / M_{\infty}\right)$, the value of $n$ indicates if the transport is Fickian diffusion or not [15].

For this data analysis, it was assumed that the 7-day cumulative fluorescence corresponded to the $\mathrm{M}_{\infty}$ value. The 7day cumulative fluorescence (adjusted, exc./emis. 370/450 $\mathrm{nm}$ ) of the leachate solutions was plotted as the log (cumulative fluorescence) vs. log (leaching time) for all sol-gel formulations, for all age periods. Units of leaching time were in minutes $(10-10,080 \mathrm{~min}$.) so that the log values $(1.0-4.0)$ would be positive for all data points. Values of $n$ were determined from the slope of the linear regression line, using the data of the first $60 \%$ of the 7-day cumulative fluorescence.

\section{RESULTS}

\subsection{Size of Pellets}

The final pellet weight (week 6) was $98 \%$ of the weight by day 17 ( 2 weeks). At 6 weeks of age, the pellet weights (from a total of 30 to 40 pellets) and dimensions (height $\times$ diameter, averages of 10 pellet samples) were: A, $0.0218 \mathrm{~g}$, $1.02 \times 3.80 \mathrm{~mm} ; \mathrm{B}, 0.0194 \mathrm{~g}, 0.97 \times 3.70 \mathrm{~mm} ; \mathrm{C}, 0.0176 \mathrm{~g}$, $0.96 \times 3.57 \mathrm{~mm}$.

\subsection{Spectra for Pyoverdin, Immobilized Pyoverdin, and Leachates}

If all the pyoverdin leached out of a microwell portion $(0.0156 \mathrm{~g})$ of the ground pyoverdin-doped sol-gel glass into $200 \mu \mathrm{l}$ of acetate buffer, the concentration of pyoverdin 
would be $5.0 \mu \mathrm{M}$. The fluorescence emission spectrum (exc. $370 \mathrm{~nm}$ ) for a $5.0 \mu \mathrm{M}$ solution of pyoverdin in $0.1 \mathrm{M}$ acetate buffer, pH 5.0 (Fig. 1a) shows there was one peak centered at $456 \mathrm{~nm}$. The emission spectrum (exc. $370 \mathrm{~nm}$ ) for a 5.0 $\mu \mathrm{M}$ solution of pyoverdin in $1 \mathrm{M} \mathrm{HCl}$ (Fig. 1b) also exhibited one peak, of greater fluorescent intensity, that was blue shifted to $436 \mathrm{~nm}$. The maximum excitation wavelength (emis. $455 \mathrm{~nm}$ ) for pyoverdin in acetate buffer was $389 \mathrm{~nm}$ (Fig. 1c), and for pyoverdin in $1 \mathrm{M} \mathrm{HCl}$ there was a double excitation peak at 367 and $378 \mathrm{~nm}$ (Fig. 1d). At an excitation of $370 \mathrm{~nm}$, the emission at $450 \mathrm{~nm}$ was about $20 \%$ higher for pyoverdin in $\mathrm{HCl}$ than for pyoverdin in acetate buffer, $\mathrm{pH}$ 5.0 .

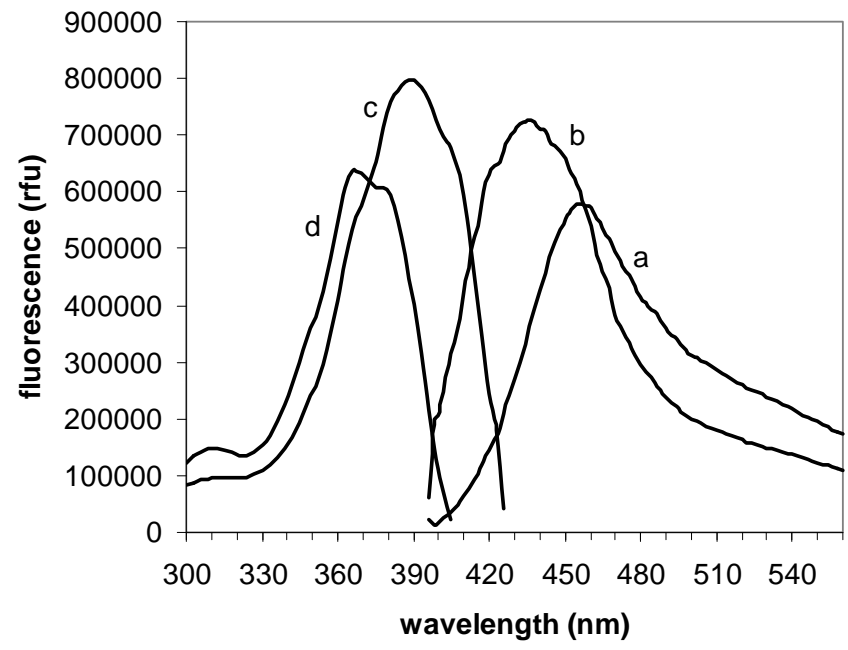

Fig. (1). Fluorescence spectra of free $5.0 \mu \mathrm{M}$ pyoverdin solutions. Emission spectra (excitation $370 \mathrm{~nm}$ ): a) pyoverdin in $0.1 \mathrm{M}$ acetate buffer, b) pyoverdin in $1.0 \mathrm{M} \mathrm{HCl}$. Excitation spectra (emission 455 $\mathrm{nm}$ ): c) pyoverdin in $0.1 \mathrm{M}$ acetate buffer, d) pyoverdin in $1.0 \mathrm{~m}$ $\mathrm{HCl}$.

In comparison, the fluorescence spectra for pyoverdin immobilized in the 3 sol-gel formulations, for all initial readings of 2-week, 4-week, and 6-week-old glass, in $0.1 \mathrm{M}$ acetate buffer, showed 2 emission peaks (Fig. 2). The larger peak was centered at about $440 \mathrm{~nm}$ and the smaller one was at $505 \mathrm{~nm}$, but as the sol-gel glasses age the height of these peaks (initial glass reading) decreased. After these glasses soaked for $10 \mathrm{~min}$. and the first leachate was removed, the spectra for the immobilized pyoverdin had only one fluorescent peak, which changed from $455 \mathrm{~nm}$ at 2 weeks (not shown), to $460-470 \mathrm{~nm}$ at 4 weeks (Fig. 3b), to $470-480 \mathrm{~nm}$ at 6 weeks (not shown). Similar results were obtained with all sol-gel formulations. At leaching times greater than 10 min. the fluorescent peak of the immobilized pyoverdin remained about the same but continued to decrease in height. For all three sol-gel formulations, the spectra of the $10 \mathrm{~min}$. leachates had 2 fluorescence peaks, centered at about $450 \mathrm{~nm}$ and $505 \mathrm{~nm}$ (Fig. 3c). The spectra for the $2^{\text {nd }}$ leachate samples (20 min.) only had one fluorescent peak, centered at about $460 \mathrm{~nm}$ at 2 weeks (not shown) and 4 weeks (Fig. 3d), and at $475 \mathrm{~nm}$ at 6 weeks (not shown). Leachate samples taken after $20 \mathrm{~min}$. also had just one peak, centered at about $460-475 \mathrm{~nm}$, but the height kept decreasing over the 7 days of leaching.
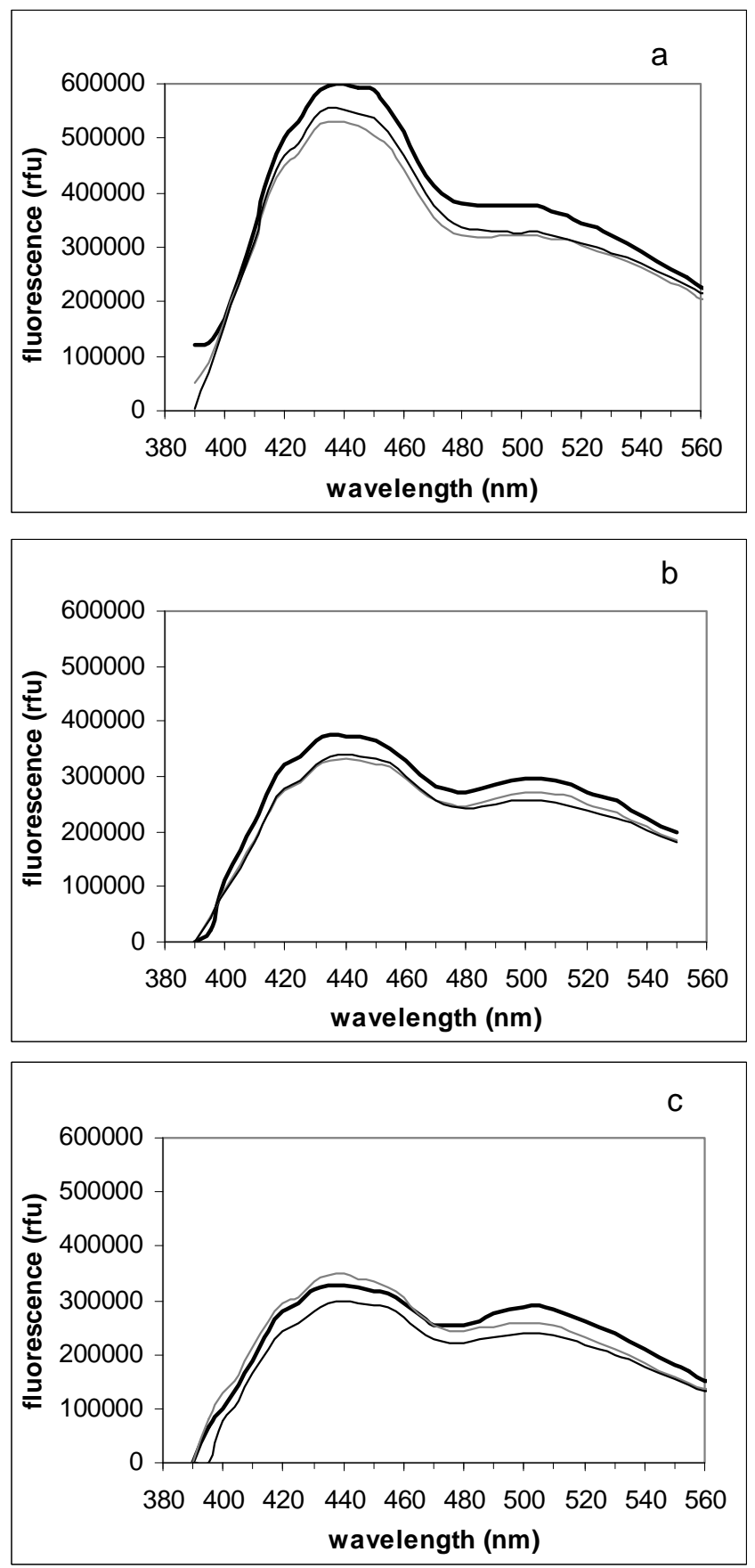

Legend: sol-gel A --, sol-gel B --, sol-gel C --.

*adjusted fluorescence, excitation $370 \mathrm{~nm}$.

Fig. (2). Fluorescence* spectra of a) 2-week-old, b) 4-week-old, and c) 6-week-old ground pyoverdin-doped sol-gel glass, when initially placed in acetate buffer.

The spectra for the 4-week-old pyoverdin-doped sol-gel glass soaked in $1 \mathrm{M} \mathrm{HCl}$ were different from the spectra seen with acetate buffer. The spectra of the initial readings of the pyoverdin-doped glass showed a main peak at about $436 \mathrm{~nm}$ and just a low shoulder at $505 \mathrm{~nm}$, for all three sol-gel formulations (Fig. 3e). After the first leachate $(10 \mathrm{~min}$.) was removed, the spectra of the immobilized pyoverdin showed the same peaks (Fig. 3f), but the peak heights decreased over 
7 days of leaching. The spectra of the first $\mathrm{HCl}$ leachate sample (10 min.) had a main peak at $436 \mathrm{~nm}$ and a low shoulder at $505 \mathrm{~nm}$ (Fig. 3g). The subsequent leachates (over 7 days) exhibited these same peaks but at lower heights, as seen in the $20 \mathrm{~min}$. $\mathrm{HCl}$ leachate (Fig. 3h).
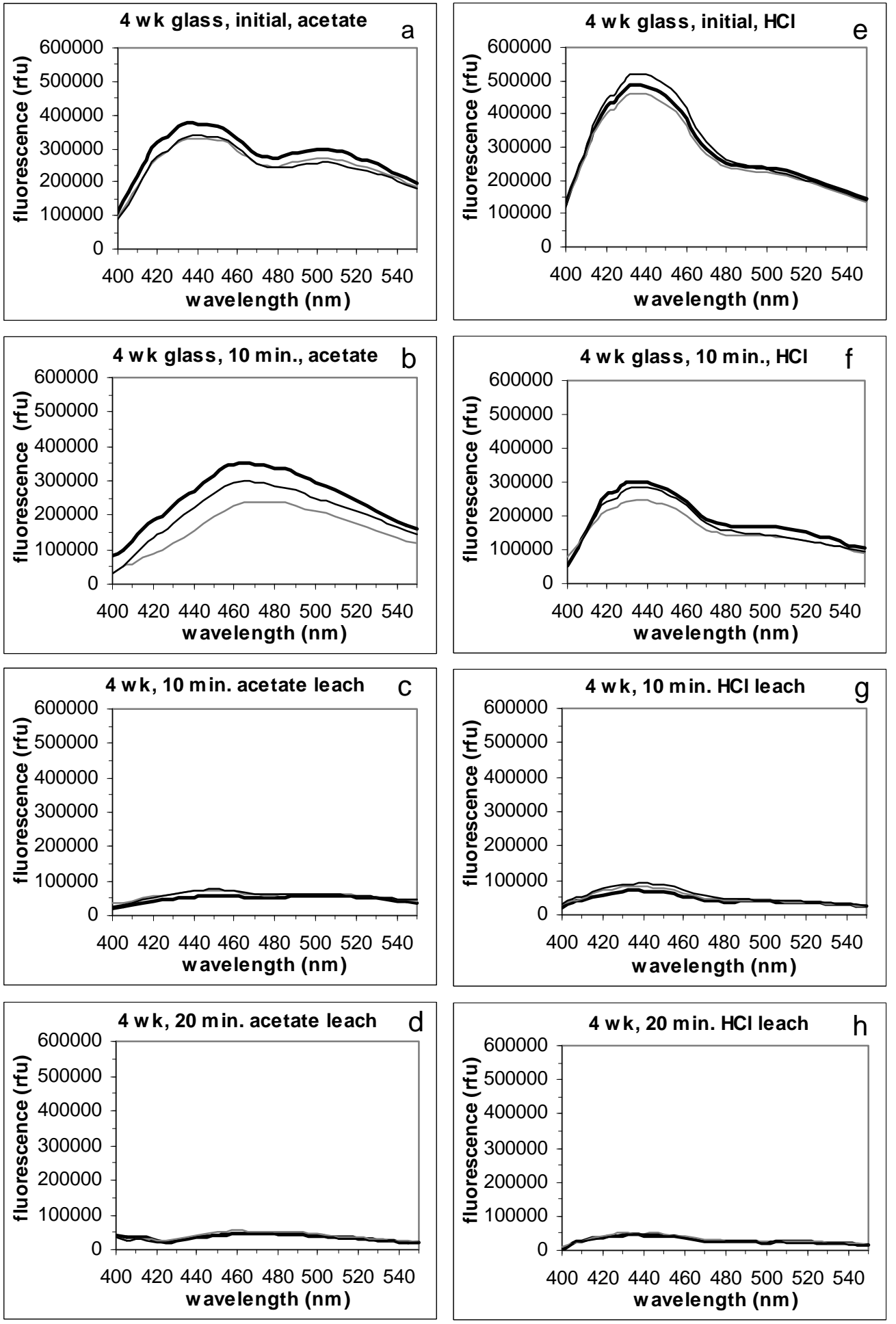

Legend: sol-gel A --, sol-gel B --, sol-gel C --.

Fig. (3). Fluorescence* spectra of 4-week-old pyoverdin-doped sol-gel glass. a) glass initially in acetate buffer, b) glass after 10 min of leaching, c) 10 min acetate leachate, d) 20 min acetate leachate, e) glass initially in $\mathrm{HCl}$, f) glass after $10 \mathrm{~min}$ of $\mathrm{HCl}$ leaching, g) $10 \mathrm{~min} \mathrm{HCl}$ leachate, h) $20 \mathrm{~min} \mathrm{HCl}$ leachate (*adj. fluor., exc. $370 \mathrm{~nm}$ ). 
showed a wider variation among sol-gel formulas, with much less leaching of pyoverdin from sol-gel A.

Values of the adjusted fluorescence (exc/emis 370/450 $\mathrm{nm}$ ) were plotted for both the ground immobilized pyoverdin and the cumulative fluorescence of the leachates, over 7 days (168 hrs.). Representative curves are shown for 4-week-old pyoverdin-doped sol-gel glass in acetate buffer in Fig. (4).

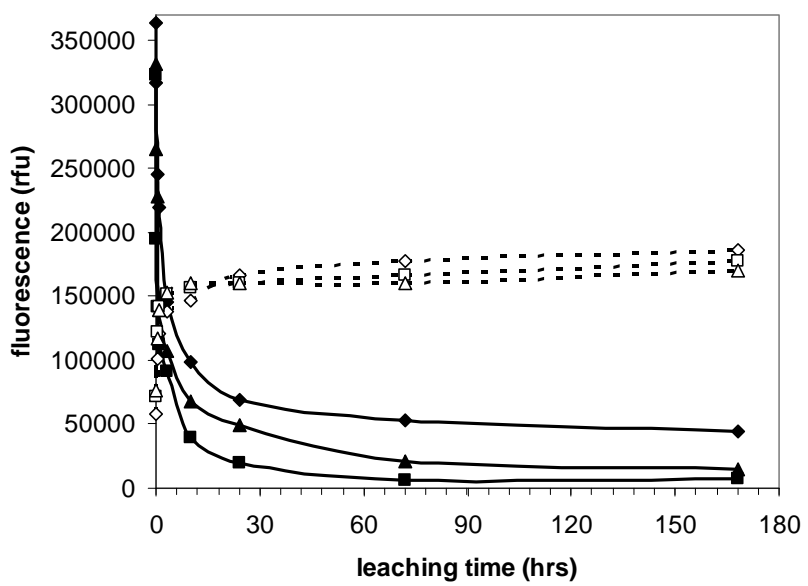

Legend: glass $\mathrm{A} \bullet-$, glass $\mathrm{B} \boldsymbol{\mathbf { }}-$, glass $\mathrm{C} \boldsymbol{\Delta} \longrightarrow$, leach $\mathrm{A} \diamond---$, leach $\mathrm{B}$ 口--- , leach $\mathrm{C} \Delta$--- .

Fig. (4). Fluorescence (adjusted, exc/emis 370/450 nm) of 4-weekold ground sol-gel glass and the cumulative leachate, over 7 days (168 hours) of leaching with acetate buffer.

\subsection{Differences in Fluorescence of Immobilized Pyoverdin and Leachates}

The data for all sol-gel formulations at all age periods (as shown in the representative curves of Fig. 4) was reduced to three fluorescence (adjusted, exc/emis 370/450 nm) values: 1. the initial fluorescence of the immobilized pyoverdin, 2 . the fluorescence of the immobilized pyoverdin after leaching for 7 days, and 3. the 7-day cumulative fluorescence of the leachate. A bar chart shows these values for ground sol-gel glasses in acetate buffer (Fig. 5). The initial fluorescence of the immobilized pyoverdin is indicated by the top of the black bar, and the fluorescence remaining after 7 days of leaching is indicated by the bottom of the black bar. The 7day (cumulative) fluorescence of the leachates is indicated by the top of the gray bars. Bars in this chart (Fig. 5) labeled with the same (lower case) letter were not significantly different $(\alpha=0.05)$, as determined from the multiple t-tests.

\subsection{Whole Pellet Experiments with Acetate Buffer}

The 7-day cumulative fluorescence of the acetate buffer leachates from whole pellets of pyoverdin-doped sol-gel glass did not vary much. The adjusted fluorescence (exc/emis 370/ 450) per nmole immobilized pyoverdin (per $200 \mu$ l leaching solution) for the 3 sol-gel formulations, at 2, 4, and $6 \mathrm{wks}$ (respectively) were: A, 266770, 222044, 222174; B, 217112, 239764, 200694; C, 233979, 263192, 204039. The 7-day cumulative fluorescence of the leachates from ground glass (Fig. 5) were between 4 - $35 \%$ lower than the corresponding fluorescence values for whole pellets (listed above).

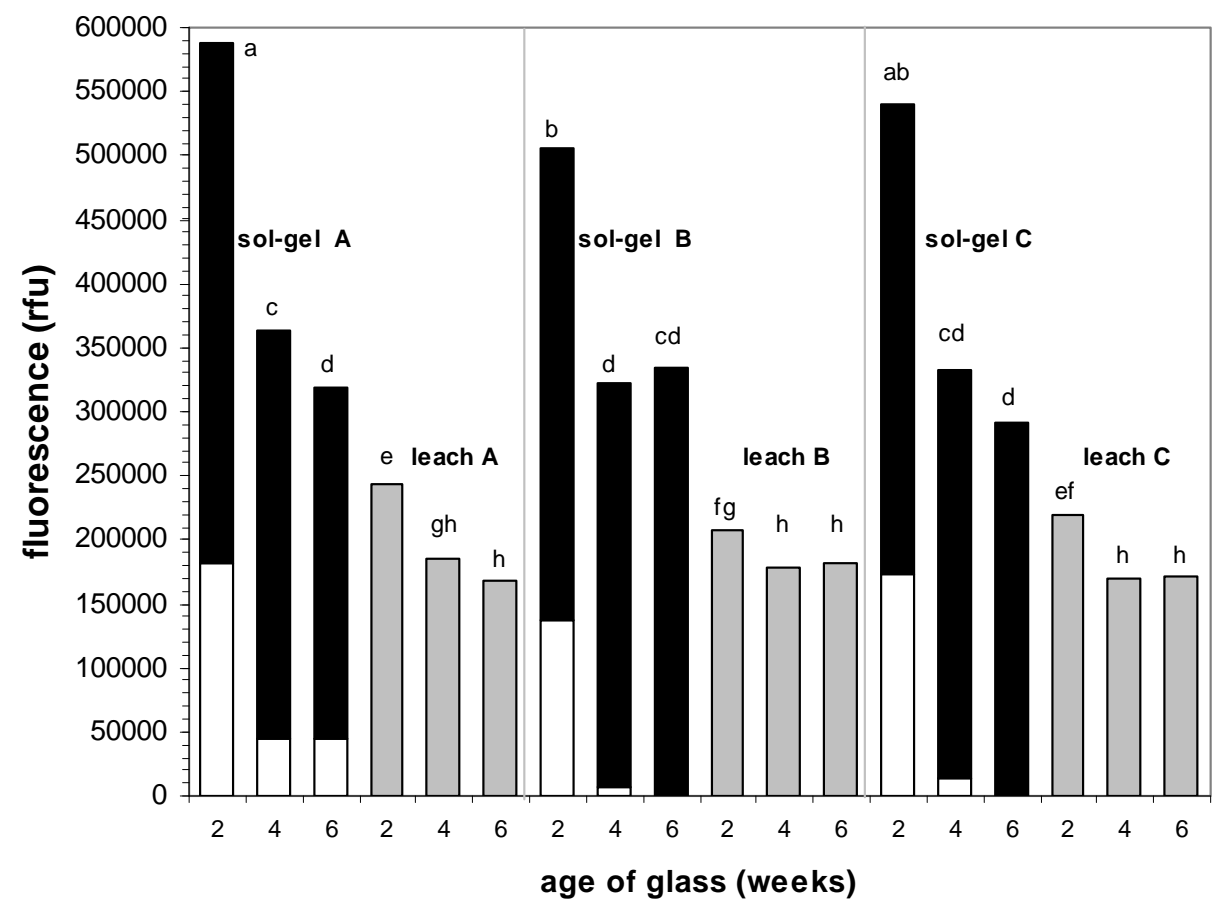

*adjusted, exc/emis 370/450 $\mathrm{nm}$.

Fig. (5). The fluorescence* of ground pyoverdin-doped sol-gel glass initially (top of black bar) and after 7 days of leaching (bottom of black bar), and the 7-day cumulative fluorescence of the leachates (top of gray bars). Data for 2-week-, 4-week-, and 6-week-old ground glasses, using $0.1 \mathrm{M}$ acetate buffer, for 3 sol-gel formulations (A, B, C). Bars labeled with the same (lower case) letter are not significantly different $(\alpha=0.05)$. 


\subsection{Leaching with $1 \mathrm{M} \mathrm{HCl}$}

Fluorescence values for 4-week-old ground pyoverdindoped sol-gel glass and the 7-day (cumulative) leachates, with $1 \mathrm{M} \mathrm{HCl}$ leaching solution, are presented (Fig. 6) in the same manner as in Fig. (5). The 7-day (cumulative) fluorescence of the $\mathrm{HCl}$ leachates from 4-week-old whole pellets is shown on the right side of Fig. (6). There was $17-22 \%$ less leaching from the ground glass than from the whole pellets.

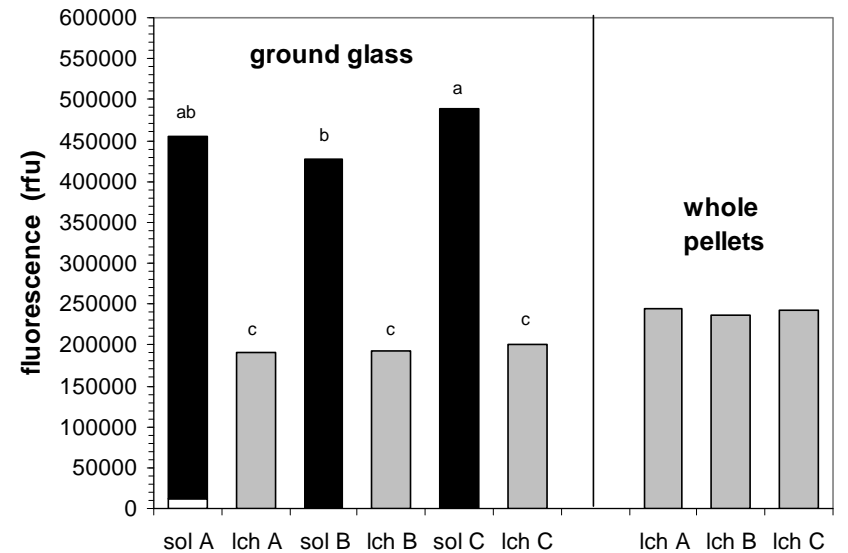

Fig. (6). Initial fluorescence* of pyoverdin-doped sol-gel glass initially (top of black bar), and after 7 days of leaching (bottom of black bar), and the 7-day cumulative fluorescence of the leachates (top of gray bars). Bars labeled with the same (lower case) letter are not significantly different $(\alpha=0.05)$. X-axis labels: sol - sol-gel glass, lch = leachate from ground sol-gel glass. *adjusted exc/emis $370 / 450 \mathrm{~nm}$.

\subsection{Leaching rate Determination}

A representative plot of log (cumulative fluorescence) vs. $\log$ (leaching time) shows the pyoverdin released (leached) from both ground and whole pellets of 6-week-old pyoverdin-doped sol-gel glass (Fig. 7). For all formulations of the ground glass, at all ages, the initial release rate $(10-20 \mathrm{~min}$.) was much faster than for the remaining (1 hr. - $168 \mathrm{hrs}$.) leaching time. For the whole pellets, the initial rate of release was only slightly faster than the remaining leaching time.

Applying equation (2) to the first $60 \%$ of the 7 -day cumulative fluorescence $\left(M_{\infty}\right.$ value) resulted in only being able to include leaching data of $10 \mathrm{~min}$. and $20 \mathrm{~min}$. for the ground glass. For the whole pellets, data up to and including 3 hours was still within $60 \%$ of the 7-day cumulative fluorescence. Values of $n$ that were calculated for the pyoverdin released (acetate leaching) from ground glass were much higher than values of $\mathrm{n}$ for whole pellets (Fig. 8). And, values of $\mathrm{n}$ for the pyoverdin released by $\mathrm{HCl}$ leaching (at 4 weeks) were also higher for ground glass (A, 0.643; B, $0.685 ; \mathrm{C}, 0.586)$ than for whole pellets $(\mathrm{A}, 0.387$; B, 0.376; $\mathrm{C}, 0.361)$.

\section{DISCUSSION}

\subsection{Influence of Immobilization Upon Pyoverdin Spectra}

As shown in Fig. (1), the single excitation peak at 389 $\mathrm{nm}$ and the single emission peak at $456 \mathrm{~nm}$ for pyoverdin in acetate buffer ( $\mathrm{pH}$ 5.0) and the double excitation peaks at
367 and $378 \mathrm{~nm}$ for pyoverdin in $1 \mathrm{M} \mathrm{HCl}(\mathrm{pH} 0.02)$ were consistent with previous results as described below. For example, using a solution of pyoverdin (from $P$. aeruginosa), Cox and Adams [17] reported exc./emis. maxima of 400/460 at neutral $\mathrm{pH}$, and double excitation peaks of 360 and 380 $\mathrm{nm}$ in acidic solution. And, these are similar to the spectra for other pyoverdins $[18,19]$.

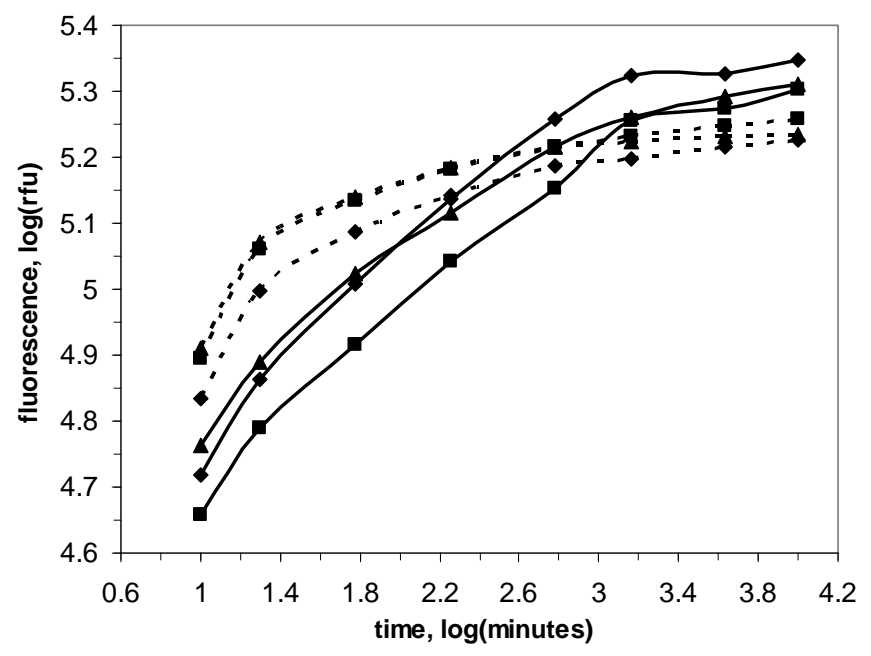

Legend: whole pellets $\mathrm{A} \bullet-$, whole pellets $\mathrm{B} \boldsymbol{-}-$, whole pellets $\mathrm{C} \boldsymbol{\Lambda}-$, ground $\mathrm{A} \diamond---$, ground $\mathrm{B} \boldsymbol{\mathbf { a }}----$, ground $\mathrm{C} \boldsymbol{\Lambda}---$.

Fig. (7). Cumulative fluorescence (exc/emis $370 / 450 \mathrm{~nm}$ ) of the acetate buffer leachate over 7 days of leaching, for both ground and whole pellets of 6-week-old pyoverdin-doped sol-gel glass. [Data points are at $10 \mathrm{~min}, 20 \mathrm{~min}, 1 \mathrm{hr}, 3 \mathrm{hrs}, 10 \mathrm{hrs}, 24 \mathrm{hrs}, 3$ days, and 7 days].

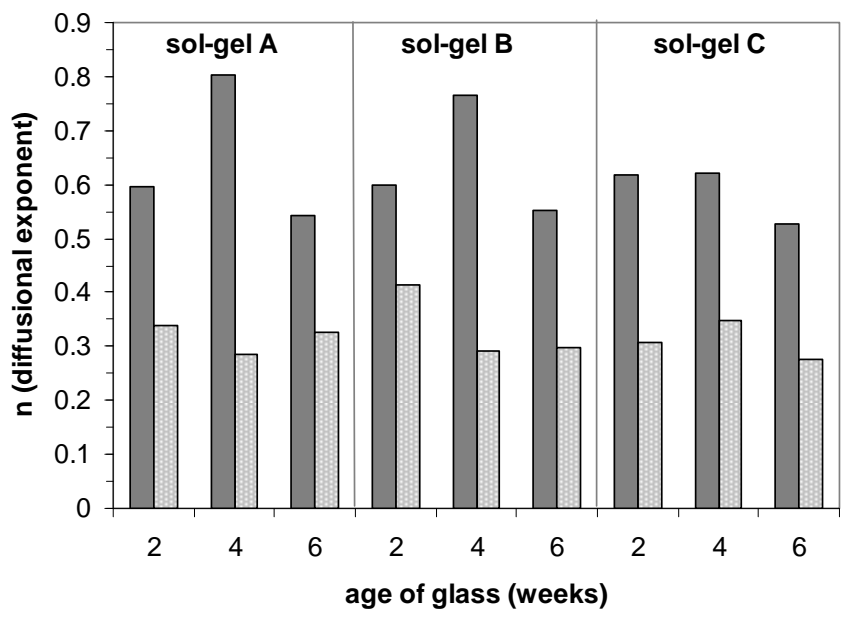

Fig. (8). Diffusional exponent, $n$, for both ground (dark) and whole (light) pellets of 2-week-, 4-week-, and 6-week-old pyoverdindoped sol-gel glass in $0.1 \mathrm{M}$ acetate buffer, $\mathrm{pH}$ 5.0.

In this study, there was a change in the spectra of pyoverdin upon immobilization. The maximum excitation was blue shifted $19 \mathrm{~nm}$, from $389 \mathrm{~nm}$ (solution) to $370 \mathrm{~nm}$ (immobilized), and the emission maximum was blue shifted $16 \mathrm{~nm}$, from $456 \mathrm{~nm}$ (solution) to $440 \mathrm{~nm}$ (immobilized, initial reading). The Stokes shift changed only slightly, from $67 \mathrm{~nm}$ (solution) to $70 \mathrm{~nm}$ (immobilized). Barrero et al. [12] 
reported that pyoverdin (in solution, from $P$. fluorescens) had exc./emis. maxima at 390/465 nm, while pyoverdin immobilized in sol-gel glass had exc./emis. maxima at 330/405 nm.

The source of the emission peak at $505 \mathrm{~nm}$, seen in both the immobilized pyoverdin and in the initial $(10 \mathrm{~min}$. leachates is uncertain. However, Keeling-Tucker and Brennan [20] noted that the emission of a fluorescent molecule doped into sol-gel glass can be complex because of the variety of interactions that the excited molecule can have with the local microenvironment. These interactions include collisions with solvent and other molecules, rotational motions, solvent dipolar relaxation, tautomerization, and protonation. The formation of an excimer or exciplex can also lead to the appearance of another emission peak at a longer wavelength [21].

It appears that only a fraction of the immobilized pyoverdin exhibited a peak at $505 \mathrm{~nm}$, because after $10 \mathrm{~min}$. of leaching the peak at $505 \mathrm{~nm}$ was absent in both the sol-gel glass (Fig. 3b) and the leachates (Fig. 3d), but the peak at approx. 455 - $470 \mathrm{~nm}$ remained. Others have also seen changes in biomolecules when immobilized in sol-gel glass. When Ferrer et al. [9] immobilized horseradish peroxidase in an acid-catalyzed TMOS sol-gel glass, they found that the small (616.5 Da) heme group leached out while none of the larger protein $(44 \mathrm{kDa})$ did.

\subsection{Influence of Aging}

For 2-week-, 4-week-, and 6-week-old sol-gel glasses, the initial fluorescence of the immobilized pyoverdin (Fig. 2) showed the same 2 peaks $(440,505 \mathrm{~nm})$. But, the initial fluorescence intensity decreased by $36-38 \%$ from 2 weeks to 4 weeks, for all three $\mathrm{R}$ values of sol-gel glass (Fig. 5). This suggested that the conformation of the pyoverdin molecule had changed, causing a change in the fluorescent yield. As the pellets aged from 2 weeks to 4 weeks, the amount of pyoverdin that leached out decreased by $14-24 \%$. As seen by the difference between the top and bottom of the black bars in Fig. (5), there was a large decrease $(68-100 \%)$ in fluorescence of immobilized pyoverdin after 7 days of leaching, for all $\mathrm{R}$ values and all ages. And, although the cumulative leachate was highest at 2 weeks (Fig. 5), the same 2week old sol-gel exhibited significantly higher pyoverdin fluorescence after the 7 days of leaching (as shown by the bottom of the black bars in Fig. 5). The practical implication for biosensor development is that a short 2 week aging period may be preferable based on the sol-gel capacity to retain pyoverdin after seven days of leaching.

Decreases (over 6 weeks) in the fluorescent intensity of the immobilized pyoverdin and in the cumulative leachate may be explained by a reduction in pore size as the sol-gel ages. From 2 weeks to 6 weeks, the average weight of the sol-gel pellets had dropped by $0.005-0.001 \mathrm{~g}$ per pellet, indicating that they were still shrinking in size, and thus the pores were also shrinking. As a sol-gel glass ages beyond the gel point, there can be changes that continue for months at room temperature [1]. These changes include more polymerization due to condensation reactions, shrinkage of the gel network (syneresis), and changes in porosity. Gill [2] believes that pore collapse (shrinkage in pores as the sol-gel ages) is the main cause of the decline in activity of encapsu- lated biomolecules. Flora and Brennan [7] found that human serum albumin that was immobilized in acid-catalyzed TMOS sol-gel glass had only $15 \%$ binding ability after the sol-gel had aged for 2 months.

\subsection{Influence of $R$ Value}

The influence of $\mathrm{R}$ value (from 5.6 - 10.8) upon the leaching behavior was minimal. In some instances, sol-gels $\mathrm{A}$ and $\mathrm{B}$ were different, as in the initial fluorescence and cumulative leachate of 2-week-old glass (Fig. 5), but in other cases sol-gels A and B were not significantly different, as in the $\mathrm{HCl}$ leaching of 4-week-old glass (Fig. 6). At the end of each 7-day leaching experiment, there was a large decrease in the fluorescence of immobilized pyoverdin for all 3 solgel formulations, as seen by the span from top to bottom of the black bars in Fig. (5). As the sol-gel glasses aged from 2 weeks to 6 weeks, the decrease in fluorescence became less, especially for sol-gel A. For the 6-week-old ground glasses, after 7 days of leaching, only in sol-gel A was there still some pyoverdin left. Thus, sol-gel A (R value 5.6) would be the best for pyoverdin retention. Using an $\mathrm{R}$ value even lower than 5.6 could possibly result in greater retention of the pyoverdin. However, it is possible that the relationship between $\mathrm{R}$ value and leaching behavior may not be linear.

Nonlinear leaching behavior has been described with TEOS sol-gel glasses. Kortesuo et al. [22] reported that an increase in the $\mathrm{R}$ value from 6 to 28 increased both the release rate and the total amount of a doped drug released from acid-catalyzed TEOS sol-gel glass. But, when others studied acid-catalyzed TEOS sol-gel glass and the range of $\mathrm{R}$ values was between 2 to 6 , an increase in $R$ value resulted in a decrease in leaching or porosity [23-25]. While an increase in $\mathrm{R}$ value can increase hydrolysis [1], the concentrations of other components, such as solvent, must also be considered. When the ratio of alcohol:TEOS was held constant, a change in the $\mathrm{R}$ value resulted in a non-linear change in the time to gel [26]. Starting at low $\mathrm{R}$ values $(<1)$, as the $\mathrm{R}$ value increased the time to gel decreased, until a certain $R$ value (dependent on the alcohol:TEOS ratio) was reached. Then, as the $\mathrm{R}$ value increased the time to gel increased.

\subsection{Influence of Leaching Solution pH}

The pyoverdin molecule, perhaps because of the alternating L- and D-amino acids in its structure, is not degraded (like other peptides are) by enzymes such as trypsin and pepsin [27], and it is resistant to denaturation by alcohol and strong acids. These properties allow a high concentration of methanol to be used in the sol-gel formulations and for $1 \mathrm{M}$ $\mathrm{HCl}$ to be used as a regenerating solution to remove iron that binds to pyoverdin when performing an iron assay.

While the largest value of initial fluorescence for the 4week-old ground glass in acetate buffer was for sol-gel A (Fig. 5), when the leaching solution was $1 \mathrm{M} \mathrm{HCl}$ the largest value of initial fluorescence was for sol-gel $\mathrm{C}$ (Fig. 6). The initial fluorescence with $\mathrm{HCl}$ was $24.7,32.4$, and $47.0 \%$ higher, for sol-gels A, B, C (respectively) than with acetate buffer. Some of this increase was expected, as the fluorescence (at exc/emis $370 / 450 \mathrm{~nm}$ ) of pyoverdin in $1 \mathrm{M} \mathrm{HCl}$ was $20 \%$ greater than the fluorescence of pyoverdin in acetate buffer (Fig. 1). The amount of $\mathrm{HCl}$ leachate from the ground glass was not significantly different $(\alpha=0.05)$ for the 
3 sol-gel formulations, and there was no difference in the $\mathrm{HCl}$ leachates from the 3 formulations of whole pellets (Fig. 6). Thus, in terms of reducing the leaching due to $1 \mathrm{M} \mathrm{HCl}$, the choice of $\mathrm{R}$ value is not important. Because the fluorescence of pyoverdin is greater in $1 \mathrm{M} \mathrm{HCl}$ than in acetate buffer, and the $\mathrm{HCl}$ leachates from the ground glasses (Fig. 6) were only slightly greater than the comparable (4 week) acetate buffer leachates (Fig. 5), it is concluded that there is no difference in the amount of leaching from $\mathrm{HCl}$ versus acetate buffer leachates.

Albrecht-Gary et al. [28] determined that the dissociation of the ferric-pyoverdin complex is complete at $\mathrm{pH}$ below 1.5. Thus, when sol-gel-immobilized pyoverdin is being used as a ferric ion biosensor, even $0.1 \mathrm{M} \mathrm{HCl}(\mathrm{pH} 1.0)$ should be effective in removing bound iron from the pyoverdin (regeneration). Although the cumulative leachate due to $1 \mathrm{M} \mathrm{HCl}$ was not different from the leachate due to acetate buffer, there might be less degradation of the sol-gel glass matrix with a milder (less acidic) regeneration. Kortesuo et al. [21] found that over the $\mathrm{pH}$ range of 1-5, the greatest degradation of TEOS sol-gel glass was at $\mathrm{pH} 1$.

\subsection{Release Mechanism}

Although the ground glasses had a greater initial leaching rate (Fig. 7) and larger $n$ (Fig. 8) than the whole pellets, it is unclear why the leachates from the ground glasses (Fig. 5) were between $4-35 \%$ less than the leachates from whole pellets (not shown). The average aspect ratio (diameter:thickness) of all the whole pellets was calculated to be 3.75. From the data of Ritger and Peppas [15] for tablets, this aspect ratio coincides with a diffusional exponent of 0.451 . Thus, since the calculated values of $\mathrm{n}$ for the whole pellets were less than 0.451 (Fig. 8), the leaching from the whole pellets deviated from Fickian diffusion. In Non-Fickian diffusion, the media penetration is in the same range as the chemical specie diffusion [21], which is undesirable if the objective is to retain the diffusing chemical. The ratio of the weight of sol-gel glass to the volume of leaching solution was constant between the ground and whole pellet experiments. But, the ratio of the surface area of the sol-gel glass to the volume of leaching solution was not. With the ground glasses, the dimensions were smaller and the surface area exposed to the leaching solution was greater, so a faster leaching rate was predicted.

The initial rate of release (between 10 and $20 \mathrm{~min}$.) of pyoverdin from the ground sol-gel glasses was very fast while the rate of release for the remaining 7 days was very slow. This was observed for all 3 ages, with both $\mathrm{HCl}$ and acetate buffer leaching, and is shown by the representative data for 6-week-old glass (Fig. 7). This agreed well with Ritger and Peppas [15], who found that a particle size distribution (such as the ground sol-gel glass) had a much faster release at early times and a much slower release at longer times, compared to the release from a monodisperse sample. And, the breadth and shape of a particle size distribution can affect the values of $n$. Kortesuo et al. [20] also reported an initial burst in the release of a doped drug from small, acidcatalyzed, TEOS sol-gel rods (made at different $\mathrm{R}$ values), and the fastest release was from the smallest sol-gel rods. For some unknown reason the values of $\mathrm{n}$ for 4 -week-old ground sol-gel glasses A and B are higher than the other values of n (Fig. 8).

For pyoverdin-doped sol-gel glass to be used as an iron biosensor in 96-well plates, the sol-gel particle size must be smaller than the whole pellets $(3.7 \mathrm{~mm}$ diameter $\times 1 \mathrm{~mm}$ height) to avoid scattering artifacts. However, larger pellets of pyoverdin-doped sol-gel glass $(3.4 \mathrm{~mm}$ diameter $\times 3.6$ $\mathrm{mm}$ height) have been successfully used in a custom-made flow cell designed the Perkin Elmer LS50B Luminescence Spectrometer [14]. If ground pyoverdin-doped sol-gel glass (of the $\mathrm{R}$ values used) was washed for 20 minutes before being used in an iron assay, the remaining activity would at least be more stable over several days than that of whole pellets.

\section{CONCLUSIONS}

While pyoverdin has been successfully doped into sol-gel glasses to produce iron biosensors by others, this study shows that there can be considerable leaching of pyoverdin from acid-catalyzed TMOS sol-gel glass. Specifically, the results from this study support the following conclusions:

1. At all $\mathrm{R}$ values, compared to the emission spectra of pyoverdin in solution, there was a change in the emission spectra (two peaks) of both immobilized pyoverdin and the first leachates. Subsequent spectra of immobilized pyoverdin and leachates only exhibited one peak. Thus, immobilization resulted in a conformational change in at least a fraction of the pyoverdin.

2. As the sol-gel glasses aged (from 2-6 weeks) there was a reduction in the initial fluorescence of the immobilized pyoverdin and a reduction in the 7-day (cumulative) leaching. And, these changes were the greatest between 2 weeks and 4 weeks.

3. The leaching behavior of pyoverdin from the ground solgel glass was different from that of the whole pellets. For the ground glass, $61 \%$ of the leaching occurred within the first 20 minutes and then the rate slowed. For the whole pellets, $54 \%$ of the leaching occurred within the first 3 hours, and the leaching rate was fairly constant over the 7 days of leaching. Although the ratio of pyoverdin-doped sol-gel glass to leaching solution was kept constant, the 7-day (cumulative) leachate for the ground glass was 4-35\% less than the leachate from whole pellets.

4. When immobilized pyoverdin was placed in $1 \mathrm{M} \mathrm{HCl}$, there was a change in the emission spectra, and these same changes were seen in the $\mathrm{HCl}$ leachates. For the 4week-old sol-gel glasses there was no difference in the amount of pyoverdin leached due to the $\mathrm{pH}$ of the leaching solution.

5. Differences in leaching due to $R$ value (5.6 to 10.8 ) were minor compared to the differences in leaching due to aging. In certain respects sol-gel A was different from solgels $\mathrm{B}$ and $\mathrm{C}$, but in other respects sol-gel $\mathrm{C}$ was different from sol-gels A and B. For 4-week-old and 6-week-old glasses, only sol-gel A still contained pyoverdin after 7 days of leaching, suggesting that it would be more suited for use in the iron biosensor, other factors being equal. 


\section{REFERENCES}

[1] Brinker CJ, Scherer GW. Sol-Gel Science: The Physics and Chemistry of Sol-Gel Processing, Academic Press, San Diego, CA, 1990.

[2] Gill I. Bio-doped nanocomposite polymers: sol-gel bioencapsulates. Chem Mater 2001; 13(10): 3404-3421.

[3] Livage J, Coradin T, Roux C. Encapsulation of biomolecules in silica gels. J Phys Condens Matter 2001; 13(33): R673-R691.

[4] Jin W, Brennan JD. Properties and applications of proteins encapsulated within sol-gel derived materials. Anal Chim Acta 2002; 461: 1-36.

[5] Yoder MF, Kisaalita WS. Fluorescence of pyoverdin in response to iron and other common well water metals. J Environ Sci Health Part A 2006; 41(3): 369-380.

[6] MacCraith BD, McDonagh C, McEvoy AK, et al. Optical chemical sensors based on sol-gel materials: Recent advances and critical issues. J Sol Gel Sci Technol 1997; 8: 1053-1061.

[7] Flora KK, Brennan JD. Effect of matrix aging on the behavior of human serum albumin entrapped in a tetraethyl orthosilicatederived glass. Chem Mater 2001; 13(11): 4170-4179.

[8] Li C-I, Lin Y-H, Shih J-P, et al. Sol-gel encapsulation of lactate dehydrogenase for optical sensing of L-lactate. Biosens Bioelectron 2002; 17: 323-330.

[9] Ferrer ML, del Monte F, Mateo J, et al. Denaturation and leaching study of horseradish peroxidase encapsulated in sol-gel matrices. J Sol Gel Sci Technol 2003; $26: 1169-1172$.

[10] Dave BC, Miller JM, Dunn B, et al. Encapsulation of proteins in bulk and thin film sol-gel matrices. J Sol Gel Sci Technol 1997; 8: 629-634.

[11] Skrdla PJ, Saavedra SS, Armstrong NR. Reduction of indicator leaching from doped sol-gels by attachment of macromolecular carriers. Appl Spectrosc 1999; 53(7): 785-791.

[12] Barrero JM, Cámara C, Pérez-Conde MC, et al. Pyoverdin-doped sol-gel glass for the spectrofluorimetric determination of iron (III). Analyst 1995; 120(2): 431-435.

[13] Fuchs R, Schäfer M, Geoffroy V, et al. Siderotyping - a powerful tool for the characterization of pyoverdines. Curr Topics Med Chem 2001; 1(1): 31-57.

[14] Yoder MF, Kisaalita WS. Specificity of an iron biosensor based on pyoverdin immobilized in sol-gel glass. J Biol Eng (Submitted 2008).
[15] Ritger PL, Peppas NA. A simple equation for description of solute release I. Fickian and non-Fickian release from non-swellable devices in the form of slabs, spheres, cylinders or discs. J Control Release 1987; 5(1): 23-36.

[16] Demange P, Wendenbaum S, Linget C, et al. Bacterial siderophores: structure and NMR assignment of pyoverdins $\mathrm{Pa}$, siderophores of Pseudomonas aeruginosa ATCC 15692. Biol Metals 1990; 3(34): $155-170$

[17] Cox CD, Adams P. Siderophore activity of pyoverdin for Pseudomonas aeruginosa. Infect Immun 1985; 48 (1): 130-138.

[18] Meyer JM, Abdallah MA. The fluorescent pigment of Pseudomonas fluorescens: biosynthesis, purification and physicochemical properties. J Gen Microbiol 1978; 107 (2): 319-328.

[19] Xiao R, Kisaalita WS. Purification of pyoverdines of Pseudomonas fluorescens $2-79$ by copper-chelate chromatography. Appl Environ Microbiol 1995; 61(11): 3769-3774.

[20] Keeling-Tucker T, Brennan JD. Fluorescent probes as reporters on the local structure and dynamics in sol-gel-derived nanocomposite materials. Chem Mater 2001; 13(10): 3331-3350.

[21] Valeur B. Molecular Fluorescence: Principles and Applications. Wiley-VCH, Weinheim, Federal Republic of Germany, 2002.

[22] Kortesuo P, Ahola M, Kangas M, et al. In vitro release of dexmedetomidine from silica xerogel monoliths: effect of sol-gel synthesis parameters. Int J Pharm 2001; 221: 107-114.

[23] Butler TM, MacCraith BD, McDonagh C. Leaching in sol-gel derived silica films for optical pH sensing. J Non Cryst Solids 1998; 224: 249-258.

[24] Noire MH, Bouzon C, Couston L, et al. Optical sensing of high acidity using a sol-gel entrapped indicator. Sensors Actuat B (Chem) 1998; 51: 214-219.

[25] McDonagh C, Bowe P, Mongey K, et al. Characterization of porosity and sensor response times of sol-gel-derived thin films for oxygen sensor applications. J Non Cryst Solids 2002; 306(2): 138-148.

[26] Klein LC. Sol-gel processing of silicates. Ann Rev Mater Sci 1985; 15(1): 227-248.

[27] Teintze M, Hossain MB, Barnes CL, et al. Structure of ferric pseudobactin, a siderophore from a plant growth promoting Pseudomonas. Biochemistry 1981; 20(22): 6446-6457.

[28] Albrecht-Gary AM, Blanc S, Rochel N, et al. Bacterial iron transport: Coordination properties of pyoverdin $\mathrm{PaA}$, a peptidic siderophore of Pseudomonas aeruginosa. Inorg Chem 1994; 33(26): 6391-6402.

(C) Yoder and Kisaalita; Licensee Bentham Open.

This is an open access article distributed under the terms of the Creative Commons Attribution License (http://creativecommons.org/license/by/2.5/), which permits unrestrictive use, distribution, and reproduction in any medium, provided the original work is properly cited. 